\title{
Synthesis and Biological Evaluation of Urea Derivatives of Combretastatins as Anticancer Agents
}

\author{
V. RAMESH ${ }^{1}$, G. PURNA CHANDER RAO ${ }^{1}$, \\ B. CHAKRAPANI ${ }^{1}$ and A. KALYAN CHAKRAVARTHY ${ }^{2 *}$ \\ ${ }^{1}$ Department of Chemistry, Acharya Nagarjuna University, Guntur, Andhra Pradesh, India \\ ${ }^{2}$ Dr. Reddy's Laboratories, Integrated Product Development, Bachupally, Hyderabad, India \\ vramesh.chem@rediffmail.com
}

Received 29 March 2018 / Accepted 20 April 2018

\begin{abstract}
A series of new combretastatin urea derivatives (12a-j) were synthesized by reacting of substituted aromatic isocyanates (11a-j) to the combretastatin pyrazole-amine. The synthesized derivatives 11a-j were evaluated for their cytotoxicity against four human cancer cell lines (breast, lung and ovarian). Among them, compounds 12a, 12c, 12d, 12f, 12g and 12i were showed potent anticancer activity.
\end{abstract}

Keywords: Combretastatin A-4, Sorafenib, Linifanib, Anticancer activity

\section{Introduction}

Combretastatin A-4 (CA-4, 1, Figure 1), which is an alkaloid extracted from the South African tree Combretum caffrum $^{1,2}$. It affects microtubule dynamics by binding to the colchicine site and shows potent cytotoxicity ${ }^{3-5}$. CA-4 also acts as a vascular disrupting agent (VDA) and induces blood flow reduction and subsequent tumor cell death ${ }^{6}$. However, its poor aqueous solubility, low bioavailability and dose-dependent toxicity restrict its clinical application ${ }^{7-9}$. Therefore to enhance these drawback the water-soluble sodium phosphate prodrug of CA-4, fosbretabulin $(\mathrm{CA}-4 \mathrm{P}, 2)$, was designed and is currently in phase II/III clinical trials ${ }^{10,11}$.

Nitrogen containing heterocycles are part of organic chemistry and plays a lead role in the biological activities. Urea was the first synthesized organic compound by Wohler in 1828 and played important roles in animal kingdom. Aryl urea moiety was a important functional group in numerous natural products and drug intermediates ${ }^{12}$ and building blocks for various heterocyclic compounds ${ }^{13,14}$. Some of the aryl urea derivatives showed a numerous biological activities such as antitumor ${ }^{15}$, inhibit the polymerization of tubulin ${ }^{16}$ anti-inflammatory ${ }^{17}$, antimalarial ${ }^{18}$ and antidiabetic ${ }^{19}$, antituberculosis, antibacterial and 
anticonvulsant $^{20-22}$ antibiotic $^{23}$ and hypoglycemic activities ${ }^{24}$. Sorafenib (3) was a bi-aryl urea (Figure 1) developed by Bayer, is currently used for the treatment of hepatocellular carcinoma $(\mathrm{HCC})^{25}$, renal cell carcinoma $(\mathrm{RCC})^{26}$. It was also showed anti-inflammatory activity ${ }^{27}$. Linifanib (4) was developed by Abbott, is used for the treatment of colorectal cancer ${ }^{28}$.

In view of above biological importance of combretastatin-A-4 and urea derivatives and continuous of efforts, we have synthesized a novel series of urea fused combretastatin derivatives and evaluated for their anticancer activity against human cancer cell lines.

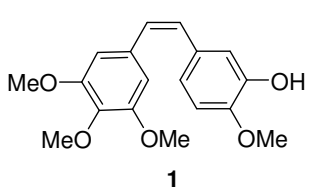<smiles>CNC(=O)c1cc(Oc2ccc(NC(=O)Nc3ccc(C(F)(F)F)c(Cl)c3)cc2)ccn1</smiles><smiles>Cc1ccc(F)c(NC(=O)Nc2ccc(-c3cccc4[nH]nc(N)c34)cc2)c1</smiles>

\section{Experimental}

Figure 1. Structure of compounds 1-4

All chemicals and reagents were obtained from Aldrich (Sigma-Aldrich, St. Louis, MO, USA), Lancaster (Alfa Aesar, Johnson Matthey Company, Ward Hill, MA, USA) and were used without further purification. Reactions were monitored by TLC, performed on silica gel glass plates containing 60 F-254 and visualization on TLC was achieved by UV light or iodine indicator. ${ }^{1} \mathrm{H}$ and ${ }^{13} \mathrm{C}$ NMR spectra were recorded on Bruker, Bruker UXNMR/XWIN-NMR (400 MHz, $300 \mathrm{MHz}$ ) instrument. Chemical shifts (d) are reported in ppm downfield from internal TMS standard. ESI spectra were recorded on Micro mass, Quattro LC using ESI+ software with capillary voltage $3.98 \mathrm{kV}$ and ESI mode positive ion trap detector. Melting points were determined with an electrothermal melting point apparatus and are uncorrected.

\section{(E)-3-(4-Methoxy-3-nitrophenyl)-2-(3,4,5-trimethoxyphenyl)acrylic acid (7)}

The compound 3,4,5-trimethoxyphenyl acetic acid (5) (7 g, $30.9 \mathrm{mmol})$ was dissolved in $20 \mathrm{~mL}$ of $\mathrm{AC}_{2} \mathrm{O}$, followed by addition of substituted 4-methoxy-3-nitrobenzaldehyde (6) (5.6 g, $30.9 \mathrm{mmol})$ and $\mathrm{Et}_{3} \mathrm{~N}(3 \mathrm{~mL})$. The reaction mixture was heated at $140{ }^{\circ} \mathrm{C}$ for 12 hours. After cooling, the reaction mixture was acidified with $35 \%$ aq $\mathrm{HCl}$. The reaction mixture was left overnight and the precipitated product was filtered. The precipitate was recrystallized from absolute ethanol afford the pure compound 7, $6.2 \mathrm{~g}$ in $52 \%$ yield. M.p: $217-218{ }^{\circ} \mathrm{C} ;{ }^{1} \mathrm{H}$ NMR (300 MHz, DMSO-d6): $\delta 3.80$ (s, 6H), 3.89 (s, 3H), 3.94 (s, 3H), 6.40 (s, 2H), 6.66 (d, 1H, $J$ $=7.3 \mathrm{~Hz}), 6.84-6.90(\mathrm{~m}, 1 \mathrm{H}), 7.16(\mathrm{~d}, 1 \mathrm{H}, J=7.3 \mathrm{~Hz}), 7.77(\mathrm{~s}, 1 \mathrm{H})$; MS (ESI): $390[\mathrm{M}+\mathrm{H}]^{+}$.

\section{(E)-Methyl 3-(4-methoxy-3-nitrophenyl)-2-(3,4,5-trimethoxyphenyl)acrylate (8)}

Concentrated $\mathrm{H}_{2} \mathrm{SO}_{4}(5 \mathrm{~mL})$ was added to a stirred solution of carboxylic acid $7(5 \mathrm{~g}$, $12.85 \mathrm{mmol})$ in absolute methanol $(40 \mathrm{~mL})$ and the mixture was heated under reflux for $6 \mathrm{~h}$. About $90 \%$ of the excess methanol was removed by evaporation, and the residue was poured into ice water $(300 \mathrm{~mL})$. The product was extracted with ether $(2 \times 40 \mathrm{~mL})$ and the combined extracts were washed with $2 \%$ aqueous $\mathrm{NaOH}$ solution $(2 \times 50 \mathrm{~mL})$ followed by water $(200 \mathrm{~mL})$. Evaporation of ether from the dried $\left(\mathrm{Na}_{2} \mathrm{SO}_{4}\right)$ solution gave the desired 
products 8, $4.9 \mathrm{~g}$ in $95 \%$ yield. ${ }^{1} \mathrm{H}$ NMR (400 MHz, DMSO-d6): $\delta 3.71(\mathrm{~s}, 3 \mathrm{H}), 3.80(\mathrm{~s}, 6 \mathrm{H})$, $3.86(\mathrm{~s}, 3 \mathrm{H}), 3.96(\mathrm{~s}, 3 \mathrm{H}), 6.43(\mathrm{~s}, 2 \mathrm{H}), 6.67(\mathrm{~d}, 1 \mathrm{H}, J=7.25 \mathrm{~Hz}), 7.77(\mathrm{~s}, 1 \mathrm{H}), 7.83-7.86(\mathrm{~m}$, 1H), 7.89-7.92 (m, 1H); MS (ESI): $404[\mathrm{M}+\mathrm{H}]^{+}$.

\section{(E)-5-(4-Methoxy-3-nitrophenyl)-4-(3,4,5-trimethoxyphenyl)-3-oxopent-4-enenitrile (9)}

Mixed (E)-methyl 3-(4-methoxy-3-nitrophenyl)-2-(3,4,5-trimethoxyphenyl)acrylate (8) (4 g, $9.92 \mathrm{mmol})$ with $\mathrm{NaH}(476 \mathrm{mg}, 19.8 \mathrm{mmol})$ in boiling tetrahydrofuran $(15 \mathrm{~mL})$ and then followed by drop wise addition of solution of acetonitrile $(5.1 \mathrm{~mL}, 9.92 \mathrm{mmol})$ in tetrahydrofuran $(10 \mathrm{~mL})$. The resulting mixture was refluxed for 4 hours and then cooling down to room temperature, after which the solution was diluted with diethylether $(15 \mathrm{~mL})$ and left to stand at room temperature for 48 hours. The precipitated sodium salt was filtered and washed with diethylether. The dry compound was dissolved in water $(5 \mathrm{~mL})$ and acidified with $\mathrm{HCl}(1 \mathrm{~mol} / \mathrm{L})$ to $\mathrm{pH} 2$. The collected extracts were crystallized from toluene, filtered and dried with $\mathrm{Na}_{2} \mathrm{SO}_{4}$ to afford pure compound 9, $3.86 \mathrm{~g}$ in $96 \%$ yield. M.p.: 220$222{ }^{\circ} \mathrm{C} ;{ }^{1} \mathrm{H}$ NMR (400 MHz, DMSO-d6): $\delta 3.81(\mathrm{~s}, 6 \mathrm{H}), 3.86(\mathrm{~s}, 3 \mathrm{H}), 3.97(\mathrm{~s}, 3 \mathrm{H}), 4.16$ (s, 2H), $6.46(\mathrm{~s}, 2 \mathrm{H}), 6.68(\mathrm{~d}, 1 \mathrm{H}, J=7.24 \mathrm{~Hz}), 7.78(\mathrm{~s}, 1 \mathrm{H}), 7.86-7.89(\mathrm{~m}, 1 \mathrm{H}), 7.93-7.95$ $(\mathrm{m}, 1 \mathrm{H}) ; \mathrm{MS}(\mathrm{ESI}): 413[\mathrm{M}+\mathrm{H}]^{+}$.

5-((E)-2-(4-Methoxy-3-nitrophenyl)-1-(3,4,5-trimethoxyphenyl)vinyl)-1H-pyrazol-3amine (10)

To a stirred mixture of compound $9(3 \mathrm{~g}, 7.28 \mathrm{mmol})$ in ethanol $(25 \mathrm{~mL})$ were added $\mathrm{NH}_{2} \mathrm{NH}_{2} . \mathrm{H}_{2} \mathrm{O}(2.7 \mathrm{~mL}, 8.73 \mathrm{mmol})$ and $\mathrm{CH}_{3} \mathrm{SO}_{3} \mathrm{H}(0.9 \mathrm{~mL}, 1.45 \mathrm{mmol})$ at room temperature and the mixture was stirred under reflux for 1 hour. The precipitate was purified by column chromatography over silica gel with ethylacetate/hexane (4:6) to afford pure compound 10, $2.7 \mathrm{~g}$ in $87 \%$ yield. M.p: $177-179{ }^{\circ} \mathrm{C} ;{ }^{1} \mathrm{H}$ NMR (400 MHz, DMSO-d6): $\delta 3.81(\mathrm{~s}, 6 \mathrm{H}), 3.86$ (s, 3H), 3.97 (s, 3H), 4.78 (s, 2H), $5.77(\mathrm{~s}, 1 \mathrm{H}), 6.46(\mathrm{~s}, 2 \mathrm{H}), 6.69(\mathrm{~d}, 1 \mathrm{H}, J=7.28 \mathrm{~Hz}), 7.78$ (s, 1H), 7.86-7.88 (m, 1H), 7.93-7.96 (m, 1H), 11.68 (s, 1H); MS (ESI): 427 [M+H] ${ }^{+}$.

\section{Procedure for synthesis of novel combretastatin urea derivatives (12a-j)}

The amine (10) $(0.46 \mathrm{mmol})$ was dissolved in $10 \mathrm{~mL}$ of dry $\mathrm{CH}_{2} \mathrm{Cl}_{2}$, followed by addition of 1-isocyanatobenzene (11a-j) $(0.46 \mathrm{mmol})$. The reaction mixture was stirred at room temperature for 12 hours. After completion of reaction product was precipitated. The precipitated product was filtered and recrystalized with ethanol to afford the pure compounds 12a-j.

1-(5-((E)-2-(4-Methoxy-3-nitrophenyl)-1-(3,4,5-trimethoxyphenyl)vinyl)-1H-pyrazol3-yl)-3-phenylurea (12a)

$81 \%$ Yield; $210-212{ }^{\circ} \mathrm{C},{ }^{1} \mathrm{H}$ NMR (400 MHz, DMSO-d6): $\delta 3.86$ (s, 3H), 3.90 (s, 6H), 3.94 $(\mathrm{s}, 3 \mathrm{H}), 5.76(\mathrm{~s}, 1 \mathrm{H}), 6.23(\mathrm{~s}, 2 \mathrm{H}), 6.78-6.84(\mathrm{~m}, 2 \mathrm{H}), 7.76-7.87(\mathrm{~m}, 3 \mathrm{H}), 7.93(\mathrm{~d}, 2 \mathrm{H}, J=$ $8.19 \mathrm{~Hz}), 8.05(\mathrm{~d}, 1 \mathrm{H}, J=7.68 \mathrm{~Hz}), 8.19(\mathrm{~s}, 1 \mathrm{H}), 9.04(\mathrm{~s}, 1 \mathrm{H}), 10.56(\mathrm{~s}, 1 \mathrm{H}), 11.67(\mathrm{~s}, 1 \mathrm{H})$, ${ }^{13} \mathrm{C}$ NMR (100 MHz, DMSO-d6): $\delta$ 57.5, 59.3, 61.5, 94.3, 115.6, 116.7, 120.7, 123.6, $127.8,129.6,130.7,131.8,133.4,138.5,140.6,140.8,141.5,142.3,143.7,150.5,153.5$, 154.6, 155.7; MS (ESI): $546[\mathrm{M}+\mathrm{H}]^{+}$.

1-(5-((E)-2-(4-Methoxy-3-nitrophenyl)-1-(3,4,5-trimethoxyphenyl)vinyl)-1H-pyrazol3-yl)-3-(3,4,5-trimethoxyphenyl)urea $(\mathbf{1 2 b})$

70\% Yield; Mp: $215-217{ }^{\circ} \mathrm{C},{ }^{1} \mathrm{H}$ NMR (400 MHz, DMSO-d6): $\delta 3.86$ (s, 3H), $3.89(\mathrm{~s}, 6 \mathrm{H})$, $3.92(\mathrm{~s}, 3 \mathrm{H}), 3.95(\mathrm{~s}, 6 \mathrm{H}), 3.97(\mathrm{~s}, 3 \mathrm{H}), 5.77(\mathrm{~s}, 1 \mathrm{H}), 6.24(\mathrm{~s}, 2 \mathrm{H}), 6.84(\mathrm{~s}, 2 \mathrm{H}), 7.76(\mathrm{~s}, 1 \mathrm{H})$, 
$7.88(\mathrm{~d}, 1 \mathrm{H}, J=8.24 \mathrm{~Hz}), 7.92(\mathrm{~d}, 1 \mathrm{H}, J=8.17 \mathrm{~Hz}), 8.19(\mathrm{~s}, 1 \mathrm{H}), 9.05(\mathrm{~s}, 1 \mathrm{H}), 10.57(\mathrm{~s}, 1 \mathrm{H})$, $11.66(\mathrm{~s}, 1 \mathrm{H}) ;{ }^{13} \mathrm{C}$ NMR (100 MHz, DMSO-d6): $\delta$ 57.5, 58.5, 59.4, 61.4, 61.9, 94.6, 112.6, $115.6,116.8,127.5,129.6,130.5,131.4,132.5,133.7,138.6,140.6,141.5,141.7,143.6$, 147.8, 150.5, 153.5, 153.9, 155.8; MS (ESI): $636[\mathrm{M}+\mathrm{H}]^{+}$.

1-(5-((E)-2-(4-Methoxy-3-nitrophenyl)-1-(3,4,5-trimethoxyphenyl)vinyl)-1H-pyrazol3-yl)-3-(4-methoxyphenyl)urea (12c)

78\% Yield.; Mp: 201-203 ${ }^{\circ} \mathrm{C},{ }^{1} \mathrm{H}$ NMR (400 MHz, DMSO-d6): $\delta 3.85$ (s, 3H), $3.88(\mathrm{~s}, 6 \mathrm{H})$, $3.91(\mathrm{~s}, 3 \mathrm{H}), 3.94(\mathrm{~s}, 3 \mathrm{H}), 5.77(\mathrm{~s}, 1 \mathrm{H}), 6.23(\mathrm{~s}, 2 \mathrm{H}), 6.80(\mathrm{~d}, 2 \mathrm{H}, J=7.65 \mathrm{~Hz}), 7.75(\mathrm{~s}, 1 \mathrm{H})$, $7.86(\mathrm{~d}, 1 \mathrm{H}, J=8.28 \mathrm{~Hz}), 7.89(\mathrm{~d}, 2 \mathrm{H}, J=7.65 \mathrm{~Hz}), 7.91(\mathrm{~d}, 1 \mathrm{H}, J=8.20 \mathrm{~Hz}), 8.19(\mathrm{~s}, 1 \mathrm{H})$, $9.06(\mathrm{~s}, 1 \mathrm{H}), 10.56(\mathrm{~s}, 1 \mathrm{H}), 11.66(\mathrm{~s}, 1 \mathrm{H}) ;{ }^{13} \mathrm{C}$ NMR (100 MHz, DMSO-d6): $\delta 56.4,57.8$, 59.6, 61.5, 94.6, 115.8, 116.7, 119.7, 122.4, 127.8, 130.4, 131.2, 133.4, 134.7, 138.5, 141.4, 141.8, 143.5, 150.4, 153.4, 153.7, 154.6, 155.9; MS (ESI): $576[\mathrm{M}+\mathrm{H}]^{+}$.

1-(4-Chlorophenyl)-3-(5-((E)-2-(4-methoxy-3-nitrophenyl)-1-(3,4,5-trimethoxyphenyl) vinyl)-1H-pyrazol-3-yl)urea (12d)

81\% Yield.; Mp: 220-222 ${ }^{\circ} \mathrm{C},{ }^{1} \mathrm{H}$ NMR (400 MHz, DMSO-d6): $\delta 3.86$ (s, 3H), $3.90(\mathrm{~s}, 6 \mathrm{H})$, $3.94(\mathrm{~s}, 3 \mathrm{H}), 5.78(\mathrm{~s}, 1 \mathrm{H}), 6.25(\mathrm{~s}, 2 \mathrm{H}), 6.85(\mathrm{~d}, 2 \mathrm{H}, J=8.23 \mathrm{~Hz}), 7.76(\mathrm{~s}, 1 \mathrm{H}), 7.87(\mathrm{~d}, 1 \mathrm{H}, J$ $=8.29 \mathrm{~Hz}), 7.90(\mathrm{~d}, 2 \mathrm{H}, J=8.23 \mathrm{~Hz}), 7.93(\mathrm{~d}, 1 \mathrm{H}, J=8.25 \mathrm{~Hz}), 8.20(\mathrm{~s}, 1 \mathrm{H}), 9.07(\mathrm{~s}, 1 \mathrm{H})$, $10.57(\mathrm{~s}, 1 \mathrm{H}), 11.67(\mathrm{~s}, 1 \mathrm{H}) ;{ }^{13} \mathrm{C}$ NMR $(100 \mathrm{MHz}, \mathrm{DMSO}-\mathrm{d} 6): \delta 57.6,59.3,61.4,94.6$, $115.6,116.7,117.9,125.7,127.7,130.5,130.7,131.3,133.5,137.6,138.4,140.5,141.4$, 141.7, 143.6, 150.5, 153.4, 153.6, 155.8; MS (ESI): $580[\mathrm{M}+\mathrm{H}]^{+}$.

1-(4-Bromophenyl)-3-(5-((E)-2-(4-methoxy-3-nitrophenyl)-1-(3,4,5-trimethoxyphenyl) vinyl)-1H-pyrazol-3-yl)urea (12e)

79\% Yield.; Mp: $225-227{ }^{\circ} \mathrm{C},{ }^{1} \mathrm{H}$ NMR (400 MHz, DMSO-d6): $\delta 3.87$ (s, 3H), $3.90(\mathrm{~s}, 6 \mathrm{H})$, $3.96(\mathrm{~s}, 3 \mathrm{H}), 5.78(\mathrm{~s}, 1 \mathrm{H}), 6.26(\mathrm{~s}, 2 \mathrm{H}), 7.76(\mathrm{~s}, 1 \mathrm{H}), 7.86(\mathrm{~d}, 1 \mathrm{H}, J=8.30 \mathrm{~Hz}), 7.90(\mathrm{~d}, 2 \mathrm{H}, J$ $=8.36 \mathrm{~Hz}), 7.94-8.04(\mathrm{~m}, 3 \mathrm{H}), 8.20(\mathrm{~s}, 1 \mathrm{H}), 9.08(\mathrm{~s}, 1 \mathrm{H}), 10.57(\mathrm{~s}, 1 \mathrm{H}), 11.67(\mathrm{~s}, 1 \mathrm{H}) ;{ }^{13} \mathrm{C}$ NMR (100 MHz, DMSO-d6): $\delta 57.6,59.7,61.4,94.7,110.6,115.6,116.7,122.5,127.6$, $130.5,131.7,132.5,133.4,138.5,138.8,140.4,141.3,141.7,143.5,150.5,153.4,153.7$, 155.9; MS (ESI): $625[\mathrm{M}+\mathrm{H}]^{+}$.

1-(4-Fluorophenyl)-3-(5-((E)-2-(4-methoxy-3-nitrophenyl)-1-(3,4,5-trimethoxyphenyl) vinyl)-1H-pyrazol-3-yl)urea (12f)

86\% Yield.; Mp: $218-220{ }^{\circ} \mathrm{C},{ }^{1} \mathrm{H}$ NMR (400 MHz, DMSO-d6): $\delta 3.86$ (s, 3H), 3.90 (s, 6H), $3.94(\mathrm{~s}, 3 \mathrm{H}), 5.77(\mathrm{~s}, 1 \mathrm{H}), 6.25(\mathrm{~s}, 2 \mathrm{H}), 7.75(\mathrm{~s}, 1 \mathrm{H}), 7.85-7.90(\mathrm{~m}, 3 \mathrm{H}), 7.94(\mathrm{~d}, 2 \mathrm{H}, J=8.35$ $\mathrm{Hz}), 7.97(\mathrm{~d}, 1 \mathrm{H}, J=8.14 \mathrm{~Hz}), 8.17(\mathrm{~s}, 1 \mathrm{H}), 9.06(\mathrm{~s}, 1 \mathrm{H}), 10.56(\mathrm{~s}, 1 \mathrm{H}), 11.66(\mathrm{~s}, 1 \mathrm{H}) ;{ }^{13} \mathrm{C}$ NMR (100 MHz, DMSO-d6): $\delta$ 57.5, 59.5, 61.9, 94.7, 115.6, 116.7, 117.8, 121.6, 127.7, $130.5,131.5,133.4,135.3,136.5,138.6,140.5,141.3,141.7,143.6,150.6,153.4,153.7$, 155.9; MS (ESI): $564[\mathrm{M}+\mathrm{H}]^{+}$.

1-(5-((E)-2-(4-Methoxy-3-nitrophenyl)-1-(3,4,5-trimethoxyphenyl)vinyl)-1H-pyrazol3-yl)-3-(4-nitrophenyl)urea (12g)

83\% Yield.; Mp: $229-231{ }^{\circ} \mathrm{C},{ }^{1} \mathrm{H}$ NMR (400 MHz, DMSO-d6): $\delta 3.87$ (s, 3H), 3.91 (s, 6H), 3.95 (s, 3H), $5.78(\mathrm{~s}, 1 \mathrm{H}), 6.26(\mathrm{~s}, 2 \mathrm{H}), 7.76(\mathrm{~s}, 1 \mathrm{H}), 7.86(\mathrm{~d}, 1 \mathrm{H}, J=8.36 \mathrm{~Hz}), 8.10-8.17(\mathrm{~m}, 3 \mathrm{H})$, 8.20-8.25 (m, 3H), 9.07 (s, 1H), 10.57 (s, 1H), 11.67 (s, 1H); ${ }^{13} \mathrm{C}$ NMR (100 MHz, DMSO-d6): $\delta 57.5,59.9,61.9,94.6,115.6,116.7,120.7,122.6,127.8,130.6,131.6,133.5,138.6,140.5$, $141.4,141.7,142.3,143.5,144.5,150.6,153.4,153.8,155.9 ;$ MS (ESI): $591[\mathrm{M}+\mathrm{H}]^{+}$. 
1-(5-((E)-2-(4-Methoxy-3-nitrophenyl)-1-(3,4,5-trimethoxyphenyl)vinyl)-1H-pyrazol3-yl)-3-(4-nitrophenyl)urea (12h)

77\% Yield.; Mp: 198-200 ${ }^{\circ} \mathrm{C},{ }^{1} \mathrm{H}$ NMR (400 MHz, DMSO-d6): $\delta 2.67$ (s, 3H), 3.85 (s, 3H), $3.90(\mathrm{~s}, 6 \mathrm{H}), 3.93(\mathrm{~s}, 3 \mathrm{H}), 5.76(\mathrm{~s}, 1 \mathrm{H}), 6.24(\mathrm{~s}, 2 \mathrm{H}), 6.85(\mathrm{~d}, 2 \mathrm{H}, J=8.32 \mathrm{~Hz}), 7.75(\mathrm{~s}, 1 \mathrm{H})$, $7.82(\mathrm{~d}, 1 \mathrm{H}, J=8.13 \mathrm{~Hz}), 7.87(\mathrm{~d}, 2 \mathrm{H}, J=8.32 \mathrm{~Hz}), 7.89(\mathrm{~d}, 1 \mathrm{H}, J=8.15 \mathrm{~Hz}), 8.18(\mathrm{~s}, 1 \mathrm{H})$, $9.05(\mathrm{~s}, 1 \mathrm{H}), 10.55(\mathrm{~s}, 1 \mathrm{H}), 11.65(\mathrm{~s}, 1 \mathrm{H}) ;{ }^{13} \mathrm{C}$ NMR (100 MHz, DMSO-d6): $\delta$ 27.4, 57.6, 59.8, 61.5, 94.5, 115.6, 116.7, 121.7, 127.4, 127.9, 130.5, 131.2, 131.7, 133.5, 138.5, 139.6, 140.5, 141.3, 141.8, 143.6, 150.7, 153.4, 153.8, 155.9; MS (ESI): $560[\mathrm{M}+\mathrm{H}]^{+}$.

1-(4-Cyanophenyl)-3-(5-((E)-2-(4-methoxy-3-nitrophenyl)-1-(3,4,5-trimethoxyphenyl) vinyl)-1H-pyrazol-3-yl)urea (12i)

68\% Yield.; Mp: $230-232{ }^{\circ} \mathrm{C},{ }^{1} \mathrm{H}$ NMR (400 MHz, DMSO-d6): $\delta 3.87$ (s, 3H), $3.92(\mathrm{~s}, 6 \mathrm{H})$, $3.94(\mathrm{~s}, 3 \mathrm{H}), 5.78(\mathrm{~s}, 1 \mathrm{H}), 6.26(\mathrm{~s}, 2 \mathrm{H}), 7.76(\mathrm{~s}, 1 \mathrm{H}), 7.82(\mathrm{~d}, 1 \mathrm{H}, J=8.24 \mathrm{~Hz}), 7.87(\mathrm{~d}, 2 \mathrm{H}, J$ $=8.40 \mathrm{~Hz}), 7.90-7.96(\mathrm{~m}, 3 \mathrm{H}), 8.20(\mathrm{~s}, 1 \mathrm{H}), 9.08(\mathrm{~s}, 1 \mathrm{H}), 10.57(\mathrm{~s}, 1 \mathrm{H}), 11.67(\mathrm{~s}, 1 \mathrm{H}) ;{ }^{13} \mathrm{C}$ NMR (100 MHz, DMSO-d6): $\delta$ 57.6, 59.6, 61.9, 94.6, 103.5, 115.7, 116.5, 120.7, 121.4, $127.8,130.2$, 131.4, 133.6, 135.6, 138.5, 140.5, 141.2, 141.5, 142.5, 143.7, 150.6, 1533.7, 153.9, 155.7; MS (ESI): $571[\mathrm{M}+\mathrm{H}]^{+}$.

1-(4-Tert-butylphenyl)-3-(5-((E)-2-(4-methoxy-3-nitrophenyl)-1-(3,4,5-

trimethoxyphenyl) vinyl)-1H-pyrazol-3-yl)urea (12j)

$70 \%$ Yield. Mp: $190-192{ }^{\circ} \mathrm{C},{ }^{1} \mathrm{H}$ NMR (400 MHz, DMSO-d6): $\delta 1.45$ (s, 9H), 3.85 (s, 3H), $3.90(\mathrm{~s}, 6 \mathrm{H}), 3.93(\mathrm{~s}, 3 \mathrm{H}), 5.76(\mathrm{~s}, 1 \mathrm{H}), 6.24(\mathrm{~s}, 2 \mathrm{H}), 7.75(\mathrm{~s}, 1 \mathrm{H}), 7.81-7.89(\mathrm{~m}, 3 \mathrm{H}), 7.92(\mathrm{~d}$, $2 \mathrm{H}, J=7.89 \mathrm{~Hz}), 7.94(\mathrm{~d}, 1 \mathrm{H}, J=8.20 \mathrm{~Hz}), 8.19(\mathrm{~s}, 1 \mathrm{H}), 9.05(\mathrm{~s}, 1 \mathrm{H}), 10.56(\mathrm{~s}, 1 \mathrm{H}), 11.65$ $(\mathrm{s}, 1 \mathrm{H}) ;{ }^{13} \mathrm{C}$ NMR (100 MHz, DMSO-d6): $\delta 33.5,36.7,57.7,59.5,61.5,94.6,115.6,116.7$, 121.6, 127.8, 128.7, 130.6, 131.4, 133.6, 138.6, 138.9, 140.6, 141.3, 141.7, 143.6, 144.6, 150.6, 153.5, 153.7, 155.7; MS (ESI): $602[\mathrm{M}+\mathrm{H}]^{+}$.

\section{Results and Discussion}

Chemistry

The general route to prepare compounds (12a-j) is described in Scheme 1. The appropriate 3,4,5-trimethoxy phenylacetic acid (5) was reacted with 4-methoxy-3-nitrobenzaldehyde (6) in acetic anhydride, triethyl amine at $140{ }^{\circ} \mathrm{C}$ for 12 hours to afford pure acrylic acid (7) in good yield. This intermediate 7 was undergoes to esterification with methanol and catalytic amount of $\mathrm{H}_{2} \mathrm{SO}_{4}$ at reflux for 6 hours to afford ester compound $\mathbf{8}$, it was reacted with $\mathrm{CH}_{3} \mathrm{CN}$ in THF, $\mathrm{NaH}$ at reflux for 4 hours to afford pure nitrile intermediate (9), this intermediate was cyclization with hydrazine hydrate in ethanol, methanesulphonic acid at reflux for 1 hours to afford pure pyrazole-amine (10) in a high yield. Finally amine intermediate $\mathbf{1 0}$ was reacted with different substituted readily available aromatic isocyanates (11a-j) in $\mathrm{CH}_{2} \mathrm{Cl}_{2}$ at room temperature for 12 hours to afford pure compounds (12a-j).

\section{Biological evaluation}

\section{In vitro cytotoxicity}

The antitumor activity of these (12a-j) compounds was screened in four cancer cell lines, namely Zr-75-1, MCF7 (human Breast cancer), A549 (human lung cancer) and A2780 (human Ovarian cancer), using sulforhodamine B (SRB) method. Adriamycin was used as a positive control. The biological data, expressed as $\mathrm{GI}_{50}$ values, are listed in Table 1. 
Interestingly, the results of this screening revealed dramatic differences in the activities of these compounds. Among them, compounds 12a, 12c, 12d, 12f, 12g and 12i were showed potent anticancer activity in all the tested cancer cell lines, with $\mathrm{GI}_{50}$ values between $0.12-89.4 \mu \mathrm{M}$, respectively.



Scheme 1. Synthesis route of compounds $12 \mathrm{a}-\mathrm{j}$

Table 1. Cytotoxic activity $\left(\mathrm{GI}_{50} \mu \mathrm{M}\right)$ of compounds $\mathbf{1 2 a}-\mathbf{j}$

\begin{tabular}{ccccc}
\hline \multirow{2}{*}{ Compound } & \multicolumn{2}{c}{ Breast cancer } & Lung cancer & Ovarian cancer \\
\cline { 2 - 5 } & Zr-75-1 & MCF7 & A549 & A2780 \\
\hline $\mathbf{1 2 a}$ & 0.23 & 0.12 & 2.34 & 1.34 \\
$\mathbf{1 2 b}$ & - & 2.11 & 1.67 & 4.78 \\
$\mathbf{1 2 c}$ & 45.2 & 1.11 & 0.34 & 34.6 \\
$\mathbf{1 2 d}$ & 1.56 & 1.89 & 3.45 & 8.23 \\
$\mathbf{1 2} \mathbf{1 2}$ & 3.67 & 12.3 & 10.7 & 25.7 \\
$\mathbf{1 2}$ & 7.80 & 1.20 & 56.2 & 9.30 \\
$\mathbf{1 2 g}$ & 2.55 & 1.55 & 3.40 & - \\
$\mathbf{1 2 h}$ & 56.2 & 89.4 & 77.4 & - \\
$\mathbf{1 2} \mathbf{1 2}$ & 44.78 & 1.22 & 1.66 & 15.4 \\
$\mathbf{1 2}$ & - & - & 17.4 & 18.9 \\
Adriamycin & 1.79 & 0.17 & 7.25 & 0.16 \\
\hline
\end{tabular}

\section{Procedure of the SRB-assay}

The synthesized compounds (12a-j) have been evaluated for their in vitro cytotoxicity in human cancer cell lines. A protocol of 48 hours continuous drug exposure has been used and a sulforhodamine B (SRB) protein assay has been used to estimate cell viability or growth. 
The cell lines were grown in DMEM medium containing 10\% fetal bovine serum and $2 \mathrm{mM}$ $L$-glutamine and were inoculated into 96 well microtiter plates in $90 \mathrm{~mL}$ at plating densities depending on the doubling time of individual cell lines. The microtiter plates were incubated at $37{ }^{\circ} \mathrm{C}, 5 \% \mathrm{CO}_{2}, 95 \%$ air and $100 \%$ relative humidity for 24 hours prior to addition of experimental drugs. Aliquots of $10 \mathrm{~mL}$ of the drug dilutions were added to the appropriate microtiter wells already containing $90 \mathrm{~mL}$ of cells, resulting in the required final drug concentrations. For each compound four concentrations $(0.1,1,10$ and $100 \mu \mathrm{M})$ were evaluated and each was done in triplicate wells. Plates were incubated further for 48 hours and assay was terminated by the addition of $50 \mathrm{~mL}$ of cold trichloroacetic acid (TCA) (final concentration, $10 \%$ TCA) and incubated for 60 minutes at $4{ }^{\circ} \mathrm{C}$. The plates were washed five times with tap water and air dried. Sulforhodamine B (SRB) solution $(50 \mathrm{~mL})$ at $0.4 \%(\mathrm{w} / \mathrm{v})$ in $1 \%$ acetic acid was added to each of the cells and plates were incubated for 20 minutes at room temperature. The residual dye was removed by washing five times with $1 \%$ acetic acid. The plates were air dried. Bound stain was subsequently eluted with $10 \mathrm{mM}$ trizma base and the absorbance was read on an ELISA plate reader at a wavelength of $540 \mathrm{~nm}$ with $690 \mathrm{~nm}$ reference wavelengths. Percent growth was calculated on a plate by plate basis for test wells relative to control wells. The above determinations were repeated three times. Percentage growth was expressed as the (ratio of average absorbance of the test well to the average absorbance of the control wells) $* 100$. Growth inhibition of $50 \%\left(\mathrm{GI}_{50}\right)$ was calculated from $[(\mathrm{Ti}-\mathrm{Tz}) /(\mathrm{C}-\mathrm{Tz})]^{*} 1001 / 450$, which is the drug concentration resulting in a $50 \%$ reduction in the net protein increase (as measured by SRB staining) in control cells during the drug incubation. Where, Tz1/4 Optical density at time zero, OD of control 1/4 C, and OD of test growth in the presence of drug $1 / 4 \mathrm{Ti}$.

\section{Conclusion}

We have synthesized a novel series of urea derivatives of combretastatins and evaluated for their in vitro anticancer activity against four human cancer cell lines Zr-75-1, MCF7 (human Breast cancer), A549 (human lung cancer) and A2780 (human Ovarian cancer). The compounds that exhibit $\mathrm{GI}_{50} \leq 10^{-5} \mathrm{M}$ are considered to be active on the respective cell lines. Among them, compounds 12a, 12c, 12d, 12f, 12g and 12i were showed potent anticancer activity in all the tested cancer cell lines, with $\mathrm{GI}_{50}$ values between $0.12-89.4 \mu \mathrm{M}$, respectively.

\section{References}

1. Pettit G R, Singh S B, Hamel E, Lin C M, Alberts D S and Garcia-Kendall D, Experientia., 1989, 45(2), 209-211; DOI:10.1007/BF01954881

2. Pettit G R, Singh S B, Boyd M R, Hamel E, Pettit R K, Schmidt J M and Hogan F, J Med Chem., 1995, 38(10), 1666-1672; DOI:10.1021/jm00010a011

3. Cirla A and Mann J, Nat Prod Rep., 2003, 20, 558-564; DOI:10.1039/B306797C

4. Nam N H, Curr Med Chem., 2003, 10(17), 1697-1722; DOI:10.2174/0929867033457151

5. Tron G C, Pirali T, Sorba G, Pagliai F, Busacca S and Genazzani A A, J Med Chem., 2006, 49(11), 3033-3044; DOI:10.1021/jm0512903

6. Nielsen T, Bentzen L, Pedersen M, Tramm T, Rijken P F, Bussink J, Horsman M R and Ostergaard L, Clin Cancer Res., 2012, 18(23), 6469; DOI:10.1158/10780432.CCR-12-2014

7. Pettit G R, Rhodes M R, Herald D L, Chaplin D J, Stratford M R, Hamel E, Pettit R K Chapuis J C and Oliva, D, Anti-Cancer Drug Des., 1998, 13(8), 981-993. 
8. Pettit G R, Toki B E, Herald D L, Boyd M R, Hamel E, Pettit R K and Chapuis J C, $J$ Med Chem., 1999, 42(8), 1459-1465; DOI:10.1021/jm9807149

9. Li Y, Luo P, Wang J, Dai J, Yang X, Wu H, Yang B and He Q, Toxicol Appl Pharmacol., 2014, 274(2), 319-327; DOI:10.1016/j.taap.2013.11.018

10. Theeramunkong S, Caldarelli A, Massarotti A, Aprile S, Caprioglio D, Zaninetti R, Teruggi A, Pirali T, Grosa G, Cesare Tron G and Genazzani A A, J Med Chem., 2011, 54(14), 4977-4986; DOI:10.1021/jm200555r

11. Zheng S, Zhong Q, Mottamal M, Zhang Q, Zhang C, LeMelle E, McFerrin H and Wang G, J Med Chem., 2014, 57(8), 3369-3381; DOI:10.1021/jm500002k

12. Tobe Y, Sasaki S I, Mizuno M, Hirose K and Neamura K, J Org Chem., 1998, 63(21), 7481-7489; DOI:10.1021/jo980441p

13. Denoyelle S, Chen T, Chen L, Wang Y B, Klosi E, Jose A H, Bertal H A and Michael C, Bioorg Med Chem Lett., 2012, 22(1), 402-409; DOI:10.1016/j.bmcl.2011.10.126

14. Marvania B, Lee P C, Chaniyara R, Dong H J, Suman S, Kakadiya R, Chou T C, Lee T C, Shah A and Su T L, Bioorg Med Chem., 2011, 19(6), 1987-1998; DOI:10.1016/j.bmc.2011.01.055

15. Yu B, Tang L D, Li Y L, Song S H, Ji X L, Lin M S and Wu C F, Bioorg Med Chem Lett., 2012, 2(1)2, 110-114; DOI:10.1016/j.bmcl.2011.11.061

16. Li Huan-Qiu, Lv Peng-Cheng, Yan Tao and Zhu Hai-Liang, Anti-Cancer Agents in Medicinal Chem., 2009, 9, 471-480.

17. Dominguéz J N, León C, Rodrigues J, Gamboa de Dominguez N, Gut J and Rosenthal P J, J Med Chem., 2005, 48(10), 3654-3658; DOI:10.1021/jm058208o

18. Audia J E, Evrard D A, Murdoch G R, Droste J J, Nissen J S, Schenck K W, Fludzinski P, Lucaites V L, Nelson D L and Cohen M L, J Med Chem., 1996, 39(14), 2773-2780; DOI:10.1021/jm960062t

19. Pluempe H and Pulls W, CA, 1971, 74, 1251154n.

20. Upadhayaya R S, Kulkarni G M, Vasireddy N R, Vandavasi J K, Dixit S S, Sharma V, Chattapadhayaya J, Bioorg Med Chem., 2009, 17(13), 4681-4692; DOI:10.1016/j.bmc.2009.04.069

21. Khan S A, Singh N and Saleem K, Eur J Med Chem., 2008, 43(10), 2272-2277; DOI:10.1016/j.ejmech.2007.12.012

22. Koçyiğit-Kaymakçıŏlu B, Rollas S, Körceğez E and Arıcıoğlu F, Eur J Pharm Sci., 2005, 26(1), 97-103; DOI:10.1016/j.ejps.2005.05.005

23. Hackbarth C J, Chen D Z and Lewis J G, Chemother., 2002, 46(9), 2752-2764; DOI:10.1128/AAC.46.9.2752-2764.2002

24. Yang J and Di R F, Chin Med J (Engl)., 2003, 116(5), 785-787.

25. Rimassa L and Santoro A, Exp Rev Anticancer Ther., 2009, 9(6), 739-745; DOI:10.1586/era.09.41

26. Wilhelm S, Carter C, Lynch M, Lowinger T, Dumas J, Smith R A, Schwartz B, Simantov R and Kelley S, Nat Rev Drug Disc., 2006, 5(10), 835-844; DOI:10.1038/nrd2130

27. Liu J Y, Park S H, Morisseau C, Hwang S H, Hammock B D and Weiss R H, Mol Cancer Ther., 2009, 8(8), 2193-2203; DOI:10.1158/1535-7163.MCT-09-0641

28. Ji ZQ J, Asma A A, Daniel H A, Jennifer J B, Peter F B, George A C, Gilbert D, Keith B G, Guo J, Christopher M H, Li J L and Patrick A M, J Med Chem., 2008, 51(5), 1231-1241; DOI:10.1021/jm701096v 